\title{
FEAL AHALYSIS
} Theory of Measure and Integration

\author{
2nd Edition
}


This page is intentionally left blank 


\title{
2nd Edition

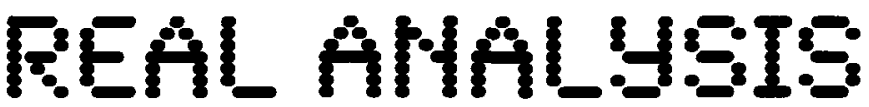 \\ Theory of Measure and Integration
}

\author{
J Yeh \\ University of California, Irvine
}




\title{
Published by
}

World Scientific Publishing Co. Pte. Ltd.

5 Toh Tuck Link, Singapore 596224

USA office: 27 Warren Street, Suite 401-402, Hackensack, NJ 07601

UK office: 57 Shelton Street, Covent Garden, London WC2H 9HE

\author{
Library of Congress Cataloging-in-Publication Data \\ Yeh, J. \\ Real analysis : theory of measure and integration / J. Yeh. -- 2nd ed. \\ p. cm. \\ Rev. ed. of: Lectures on real analysis. 1st ed. c2000. \\ ISBN 981-256-653-8 (alk. paper) -- ISBN 981-256-654-6 (pbk. : alk. paper) \\ 1. Measure theory. 2. Lebesgue integral. 3. Integrals, Generalized. 4. Mathematical \\ analysis. 5. Lp spaces. I. Yeh, J. Lectures on real analysis. II. Title.
}

QA312.Y44 2006

$515^{\prime} \cdot 42--d c 22$

2006045274

\section{British Library Cataloguing-in-Publication Data}

A catalogue record for this book is available from the British Library.

Copyright (C) 2006 by World Scientific Publishing Co. Pte. Ltd.

All rights reserved. This book, or parts thereof, may not be reproduced in any form or by any means, electronic or mechanical, including photocopying, recording or any information storage and retrieval system now known or to be invented, without written permission from the Publisher.

For photocopying of material in this volume, please pay a copying fee through the Copyright Clearance Center, Inc., 222 Rosewood Drive, Danvers, MA 01923, USA. In this case permission to photocopy is not required from the publisher.

Printed in Singapore by Mainland Press 


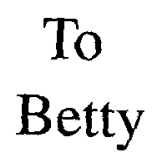


This page is intentionally left blank 


\section{Contents}

Preface to the First Edition xiii

Preface to the Second Edition xvii

Notations $\quad$ xix

1 Measure Spaces 1

$\S 0$ Introduction $\ldots \ldots \ldots \ldots \ldots \ldots \ldots \ldots \ldots \ldots \ldots$

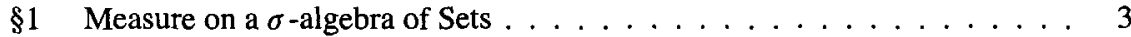

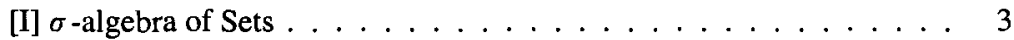

[II] Limits of Sequences of Sets . . . . . . . . . . . . . . . . 4

[III] Generation of $\sigma$-algebras $\ldots \ldots \ldots \ldots \ldots \ldots \ldots$

[IV] Borel $\sigma$-algebras . . . . . . . . . . . . . . . . 9

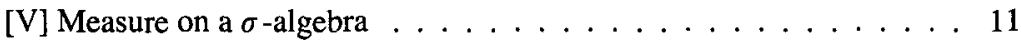

[VI] Measures of a Sequence of Sets . . . . . . . . . . . . 14

[VII] Measurable Space and Measure Space . . . . . . . . . . . . . . 17

[VIII] Measurable Mapping . . . . . . . . . . . . . . . . . . . . . . . . 19

[IX] Induction of Measure by Measurable Mapping . . . . . . . . . 22

$\$ 2$ Outer Measures . . . . . . . . . . . . . . . . . . . 28

[I] Construction of Measure by Means of Outer Measure . . . . . . . 28

[II] Regular Outer Measures . . . . . . . . . . . . . . . . . . . . . . 32

[III] Metric Outer Measures . . . . . . . . . . . . . . . . . . 34

[IV] Construction of Outer Measures . . . . . . . . . . . . . . 37

$\$ 3$ Lebesgue Measure on $\mathbb{R} \ldots \ldots \ldots \ldots \ldots \ldots$

[I] Lebesgue Outer Measure on $\mathbb{R}$. . . . . . . . . . . . . . . . . . 41

[II] Some Properties of the Lebesgue Measure Space . . . . . . . . . . 45

[III] Existence of Non Lebesgue Measurable Sets . . . . . . . . . . . 49

[IV] Regularity of Lebesgue Outer Measure . . . . . . . . . . 51

[V] Lebesgue Inner Measure on $\mathbb{R} \ldots \ldots$

$\$ 4$ Measurable Functions $\ldots \ldots \ldots \ldots \ldots \ldots$

[I] Measurability of Functions . . . . . . . . . . . . 70

[II] Operations with Measurable Functions . . . . . . . . . . . . 74

[III] Equality Almost Everywhere . . . . . . . . . . . . . . . . 78

[IV] Sequence of Measurable Functions . . . . . . . . . . . . 79

[V] Continuity and Borel and Lebesgue Measurability of Functions on $\mathbb{R} \quad 83$ 
[VI] Cantor Ternary Set and Cantor-Lebesgue Function . . . . . . . . . . 85

$\$ 5$ Completion of Measure Space . . . . . . . . . . . . . . . . . 95

[I] Complete Extension and Completion of a Measure Space . . . . . . 95

[II] Completion of the Borel Measure Space to the Lebesgue Measure Space . . . . . . . . . . . . . . . . . . 98

$\S 6$ Convergence a.e. and Convergence in Measure . . . . . . . . . . . . 100

[I] Convergence a.e. . . . . . . . . . . . . . . . . . . . 100

[II] Almost Uniform Convergence . . . . . . . . . . . . . . . . . . . . . . . . . . . . . . . . . . . . . . . .

[III] Convergence in Measure . . . . . . . . . . . . . . . . . . 107

[IV] Cauchy Sequences in Convergence in Measure . . . . . . . . . . . 112

[V] Approximation by Step Functions and Continuous Functions . . . . 115

2 The Lebesgue Integral 127

$\$ 7$ Integration of Bounded Functions on Sets of Finite Measure . . . . . . . 127

[I] Integration of Simple Functions . . . . . . . . . . . . . . . 127

[II] Integration of Bounded Functions on Sets of Finite Measure . . . . . 131

[III] Riemann Integrability . . . . . . . . . . . . . . . . . . . 140

$\S 8$ Integration of Nonnegative Functions . . . . . . . . . . . . . . . . 152

[I] Lebesgue Integral of Nonnegative Functions . . . . . . . . . . . . . 152

[II] Monotone Convergence Theorem . . . . . . . . . . . . . . . . . 154

[III] Approximation of the Integral by Truncation . . . . . . . . . . . 162

$\S 9$ Integration of Measurable Functions . . . . . . . . . . . . . . . . 169

[I] Lebesgue Integral of Measurable Functions . . . . . . . . . . . . . . . . . . . . . . . . . . . . . . . . .

[II] Convergence Theorems . . . . . . . . . . . . . . . . . . 178

[III] Convergence Theorems under Convergence in Measure . . . . . . . 182

[IV] Approximation of the Integral by Truncation . . . . . . . . . . . 183

[V] Translation and Linear Transformation in the Lebesgue Integral on $\mathbb{R} 189$

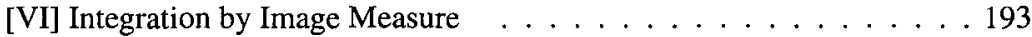

$\S 10$ Signed Measures . . . . . . . . . . . . . . . . . . . . 202

[I] Signed Measure Spaces . . . . . . . . . . . . . . . . . . . . . . . . . . 202

[II] Decomposition of Signed Measures . . . . . . . . . . . . . . . . . . . . . . . . . . . . . .

[III] Integration on a Signed Measure Space . . . . . . . . . . . . . 217

$\$ 11$ Absolute Continuity of a Measure . . . . . . . . . . . . . . . . . . . . 224

[I] The Radon-Nikodym Derivative . . . . . . . . . . . . . . . 224

[II] Absolute Continuity of a Signed Measure Relative to a Positive Measure ... . . . . . . . . . . . . . . 225

[III] Properties of the Radon-Nikodym Derivative . . . . . . . . . . . 236

3 Differentiation and Integration 245

$\S 12$ Monotone Functions and Functions of Bounded Variation . . . . . . . . . . 245

[I] The Derivative . . . . . . . . . . . . . . . . . . . 245

[II] Differentiability of Monotone Functions . . . . . . . . . . . . . . 251

[III] Functions of Bounded Variation . . . . . . . . . . . . . . . . . 261

$\$ 13$ Absolutely Continuous Functions . . . . . . . . . . . . . . . . 270

[I] Absolute Continuity . . . . . . . . . . . . . . . . 270 
[II] Banach-Zarecki Criterion for Absolute Continuity . . . . . . . . 273

[III] Singular Functions . . . . . . . . . . . . . . . . . 276

[IV] Indefinite Integrals . . . . . . . . . . . . . . . . 276

[V] Calculation of the Lebesgue Integral by Means of the Derivative . 287

[VI] Length of Rectifiable Curves . . . . . . . . . . . . . . . . 298

$\S 14$ Convex Functions . . . . . . . . . . . . . . . . . . . . 308

[I] Continuity and Differentiability of a Convex Function . . . . . . 308

[II] Monotonicity and Absolute Continuity of a Convex Function . . . 317

[III] Jensen's Inequality . . . . . . . . . . . . . . . . 320

4 The Classical Banach Spaces 323

$\$ 15$ Normed Linear Spaces . . . . . . . . . . . . . . . . . . . . 323

[I] Banach Spaces . . . . . . . . . . . . . . . . . . . . . . . 323

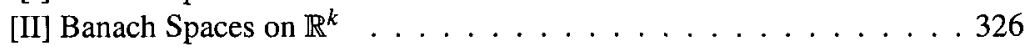

[III] The Space of Continuous Functions $C([a, b]) \ldots \ldots \ldots \ldots . \ldots 329$

[IV] A Criterion for Completeness of a Normed Linear Space . . . . . 331

[V] Hilbert Spaces . . . . . . . . . . . . . . . . . . . . . 333

[VI] Bounded Linear Mappings of Normed Linear Spaces . . . . . . . . 334

[VII] Baire Category Theorem . . . . . . . . . . . . . . . . . . . . . 344

[VIII] Uniform Boundedness Theorems . . . . . . . . . . . . . . 347

[IX] Open Mapping Theorem . . . . . . . . . . . . . . . . . . . 350

[X] Hahn-Banach Extension Theorems . . . . . . . . . . . . 357

[XI] Semicontinuous Functions . . . . . . . . . . . . . . 370

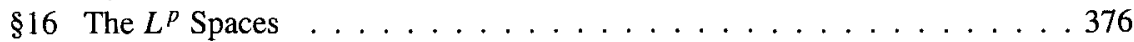

[I] The $\mathcal{L}^{p}$ Spaces for $p \in(0, \infty) \ldots \ldots \ldots \ldots \ldots \ldots$

[II] The Linear Spaces $\mathcal{L}^{p}$ for $p \in[1, \infty) \ldots \ldots \ldots . \ldots 379$

[III] The $L^{p}$ Spaces for $p \in[1, \infty) \ldots \ldots \ldots \ldots . \ldots \ldots 34$

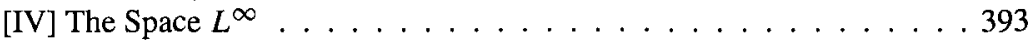

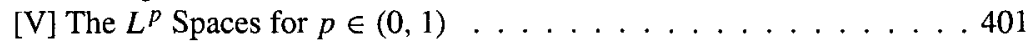

[VI] Extensions of Hölder's Inequality . . . . . . . . . . . . . 406

$\$ 17$ Relation among the $L^{p}$ Spaces . . . . . . . . . . . . . . 412

[I] The Modified $L^{p}$ Norms for $L^{p}$ Spaces with $p \in[1, \infty] \ldots \ldots 412$

[II] Approximation by Continuous Functions . . . . . . . . . . . . . 414

[III] $L^{p}$ Spaces with $p \in(0,1] \ldots \ldots \ldots \ldots \ldots$. . . . . . . . . . . .

[IV] The $\ell^{p}$ Spaces . . . . . . . . . . . . . . . . . . 422

$\$ 18$ Bounded Linear Functionals on the $L^{p}$ Spaces $\ldots \ldots \ldots$. . . . . . . 429

[1] Bounded Linear Functionals Arising from Integration . . . . . . . 429

[II] Approximation by Simple Functions . . . . . . . . . . . 432

[III] A Converse of Hölder's Inequality . . . . . . . . . . . . . . 434

[IV] Riesz Representation Theorem on the $L^{p}$ Spaces . . . . . . . . . . 437

$\$ 19$ Integration on Locally Compact Hausdorff Space . . . . . . . . . . . . . 445

[I] Continuous Functions on a Locally Compact Hausdorff Space . . 445

[II] Borel and Radon Measures . . . . . . . . . . . . . . . . . 450

[III] Positive Linear Functionals on $C_{c}(X) \ldots \ldots \ldots \ldots . \ldots 45$

[IV] Approximation by Continuous Functions . . . . . . . . . . 463 
[V] Signed Radon Measures . . . . . . . . . . . . . . . . . . . . . 467

[VI] The Dual Space of $C(X) \ldots \ldots \ldots \ldots$. . . . . . . . 471

5 Extension of Additive Set Functions to Measures 481

$\$ 20$ Extension of Additive Set Functions on an Algebra . . . . . . . . . . 481

[I] Additive Set Function on an Algebra . . . . . . . . . . . . 481

[II] Extension of an Additive Set Function on an Algebra to a Measure . 486

[III] Regularity of an Outer Measure Derived from a Countably Additive Set Function on an Algebra . . . . . . . . . . . . . . . . 486

[IV] Uniqueness of Extension of a Countably Additive Set Function on an Algebra to a Measure . . . . . . . . . . . . . . . . 489

[V] Approximation to a $\sigma$-algebra Generated by an Algebra . . . . . . 491

[VI] Outer Measure Based on a Measure . . . . . . . . . . . . . . . . 494

$\$ 21$ Extension of Additive Set Functions on a Semialgebra . . . . . . . . 496

[1] Semialgebras of Sets . . . . . . . . . . . . . . . . . . 496

[II] Additive Set Function on a Semialgebra . . . . . . . . . . . . 498

[III] Outer Measures Based on Additive Set Functions on a Semialgebra . 502

$\$ 22$ Lebesgue-Stieltjes Measure Spaces . . . . . . . . . . . . . . . . . 505

[I] Lebesgue-Stieltjes Outer Measures . . . . . . . . . . . . . . . . 505

[II] Regularity of the Lebesgue-Stieltjes Outer Measures . . . . . . . . . 509

[III] Absolute Continuity and Singularity of a Lebesgue-Stieltjes Measure 511

[IV] Decomposition of an Increasing Function . . . . . . . . . . . . 519

$\$ 23$ Product Measure Spaces . . . . . . . . . . . . . . . . . . . . . . 527

[I] Existence and Uniqueness of Product Measure Spaces . . . . . . . . 527

[II] Integration on Product Measure Space . . . . . . . . . . . . . . 531

[III] Completion of Product Measure Space . . . . . . . . . . . 543

[IV] Convolution of Functions . . . . . . . . . . . . . . . . 547

[V] Some Related Theorems . . . . . . . . . . . . . . . . . . . . . 587

6 Measure and Integration on the Euclidean Space 597

$\$ 24$ Lebesgue Measure Space on the Euclidean Space . . . . . . . . . . . 597

[I] Lebesgue Outer Measure on the Euclidean Space . . . . . . . 597

[II] Regularity Properties of Lebesgue Measure Space on $\mathbb{R}^{n} \ldots \ldots$. . . 602

[III] Approximation by Continuous Functions . . . . . . . . . . . . 605

[IV] Lebesgue Measure Space on $\mathbb{R}^{n}$ as the Completion of a Product

Measure Space . . . . . . . . . . . . . . . . . . . . . 609

[V] Translation of the Lebesgue Integral on $\mathbb{R}^{n} \ldots \ldots$. . . . . . 610

[VI] Linear Transformation of the Lebesgue Integral on $\mathbb{R}^{n} \ldots \ldots$. . . 612

$\$ 25$ Differentiation on the Euclidean Space . . . . . . . . . . . . 620

[I] The Lebesgue Differentiation Theorem on $\mathbb{R}^{n} \ldots \ldots$. . . . . . 620

[II] Differentiation of Set Functions with Respect to the Lebesgue Measure . . . . . . . . . . . . . . . . . 632

[III] Differentiation of the Indefinite Integral . . . . . . . . . . . 634

[IV] Density of Lebesgue Measurable Sets Relative to the Lebesgue Measure . . . . . . . . . . . . . . .635 
[V] Signed Borel Measures on $\mathbb{R}^{n} \ldots \ldots \ldots$. . . . . . . . . . 641

[VI] Differentiation of Borel Measures with Respect to the Lebesgue

Measure . . . . . . . . . . . . . . . . . . . . . . . 643

$\$ 26$ Change of Variable of Integration on the Euclidean Space . . . . . . . 649

[I] Change of Variable of Integration by Differentiable Transformations 649

[II] Spherical Coordinates in $\mathbb{R}^{n} \ldots$. . . . . . . . . . . . . 661

[III] Integration by Image Measure on Spherical Surfaces . . . . . . . 667

7 Hausdorff Measures on the Euclidean Space 675

$\$ 27$ Hausdorff Measures . . . . . . . . . . . . . . . . . . . . . .675

[I] Hausdorff Measures on $\mathbb{R}^{n} \ldots \ldots$. . . . . . . . . . . . 675

[II] Equivalent Definitions of Hausdorff Measure . . . . . . . . . . 680

[III] Regularity of Hausdorff Measure . . . . . . . . . . . . . . . 686

[IV] Hausdorff Dimension . . . . . . . . . . . . . . . . . . . . 689

$\$ 28$ Transformations of Hausdorff Measures . . . . . . . . . . . . . . . . 694

[I] Hausdorff Measure of Transformed Sets . . . . . . . . . . . . 694

[II] 1-dimensional Hausdorff Measure . . . . . . . . . . . . . . 699

[III] Hausdorff Measure of Jordan Curves . . . . . . . . . . . . . . . 700

$\$ 29$ Hausdorff Measures of Integral and Fractional Dimensions . . . . . . . 705

[I] Hausdorff Measure of Integral Dimension and Lebesgue Measure . . 705

[II] Calculation of the $n$-dimensional Hausdorff Measure of a Unit Cube in $\mathbb{R}^{n} \ldots \ldots \ldots$. . . . . . . . . . . . . . . 707

[III] Transformation of Hausdorff Measure of Integral Dimension . . . . . 713

[IV] Hausdorff Measure of Fractional Dimension . . . . . . . . 718

$\begin{array}{ll}\text { Bibliography } & \mathbf{7 2 7}\end{array}$

$\begin{array}{ll}\text { Index } & 729\end{array}$ 
This page is intentionally left blank 


\section{Preface to the First Edition}

This monograph evolved from a set of lecture notes for a course entitled Real Analysis that I taught at the University of California, Irvine. The subject of this course is the theory of measure and integration. Its prerequisite is advanced calculus. All of the necessary background material can be found, for example, in R. C. Buck's Advanced Calculus. The course is primarily for beginning graduate students in mathematics but the audience usually includes students from other disciplines too. The first five chapters of this book contain enough material for a one-year course. The remaining two chapters take an academic quarter to cover.

Measure is a fundamental concept in mathematics. Measures are introduced to estimate sizes of sets. Then measures are used to define integrals. Here is an outline of the book.

Chapter 1 introduces the concepts of measure and measurable function. $\$ 1$ defines measure as a nonnegative countably additive set function on a $\sigma$-algebra of subsets of an arbitrary set. Measurable mapping from a measure space into another is then defined. $\$ 2$ presents construction of a measure space by means of an outer measure. To have a concrete example of a measure space early on, the Lebesgue measure space on the real line $\mathbb{R}$ is introduced in \$3. Subsequent developments in the rest of Chapter 1 and Chapter 2 are in the setting of a general measure space. (This is from the consideration that in the definition of a measure and an integral with respect to a measure the algebraic and topological structure of the underlying space is irrelevant and indeed unnecessary. Topology of the space on which a measure is defined becomes relevant when one considers the regularity of the measure, that is, approximation of measurable sets by Borel sets.) $\$ 4$ treats measurable functions, in particular algebraic operations on measurable functions and pointwise limits of sequences of measurable functions. $\$ 5$ shows that every measure space can be completed. $\$ 6$ compares two modes of convergence of a sequence of measurable functions: convergence almost everywhere and convergence in measure. The Borel-Cantelli Lemma and its applications are presented. A unifying theorem (Theorem 6.5 ) is introduced from which many other convergence theorems relating the two modes of convergence are derived subsequently. These include Egoroff's theorem on almost uniform convergence, Lebesgue's and Riesz's theorems.

Chapter 2 treats integration of functions on an arbitrary measure space. In $\S 7$ the Lebesgue integral, that is, an integral with respect to a measure, is defined for a bounded real-valued measurable function on a set of finite measure. The Bounded Convergence Theorem on the commutation of integration and limiting process for a uniformly bounded 
sequence of measurable functions which converges almost everywhere on a set of finite measure is proved here. The proof is based on Egoroff's theorem. On the Lebesgue measure space on $\mathbb{R}$, comparison of the Lebesgue integral and the Riemann integral is made. $\$ 8$ contains the fundamental idea of integration with respect to a measure. It is shown here that for every nonnegative extended real-valued measurable function on a measurable set the integral with respect to the measure always exists even though it may not be finite. The Monotone Convergence Theorem for an increasing sequence of nonnegative measurable functions, the most fundamental of all convergence theorems regarding commutation of integration and convergence of the sequence of integrands, is proved here. Fatou's Lemma concerning the limit inferior of a sequence of nonnegative measurable functions is derived from the Monotone Convergence Theorem. In $\$ 9$ the integral of an extended real-valued measurable function on a measurable set is then defined as the difference of the integrals of the positive and negative parts of the function provided the difference exists in the extended real number system. The generalized monotone convergence theorem for a monotone sequence of extended real-valued measurable functions, generalized Fatou's lemma for the limit inferior and the limit superior of a sequence of extended real-valued measurable functions, and Lebesgue's Dominated Convergence Theorem are proved here. Fatou's Lemma and Lebesgue's Dominated Convergence Theorem under convergence in measure are included. In $\$ 10$ a signed measure is defined as an extended real-valued countably additive set function on a $\sigma$-algebra and then shown to be the difference of two positive measures. In $\S 11$ the Radon-Nikodym derivative of a signed measure with respect to a positive measure is defined as a function which we integrate with respect to the latter to obtain the former. The existence of the Radon-Nikodym derivative is then proved under the assumption that the former is absolutely continuous with respect to the latter and that both are $\sigma$-finite. (The fact that the Radon-Nikodym derivative is a derivative not only in name but in fact it is the derivative of a measure with respect to another is shown for Borel measures on the Euclidean space in $\$ 25$.)

Chapter 3 treats the interplay between integration and differentiation on the Lebesgue measure space on $\mathbb{R}$. $\$ 12$ presents Lebesgue's theorem that every real-valued increasing function on $\mathbb{R}$ is differentiable almost everywhere on $\mathbb{R}$. The proof is based on a Vitali covering theorem. This is followed by Lebesgue's theorem on the integral of the derivative of a real-valued increasing function on a finite closed interval in $\mathbb{R}$. Functions of bounded variation are included here. $\$ 13$ defines absolute continuity of a real-valued function on a finite closed interval in $\mathbb{R}$ and then shows that a function is absolutely continuous if and only if it is an indefinite integral of a Lebesgue integrable function. This is followed by Lebesgue's decomposition of a real-valued increasing function as the sum of an absolutely continuous function and a singular function. Such methods of calculating a Riemann integral in calculus as the Fundamental Theorem of Calculus, integration by parts, and change of variable of integration find their counterparts in the Lebesgue integral here. $\$ 14$ treats convex functions and in particular their differentiability and absolute continuity property. Jensen's inequality is included here.

Chapter 4 treats the $L^{p}$ spaces of measurable functions $f$ with integrable $|f|^{p}$ for $p \in$ $(0, \infty)$ and the space $L^{\infty}$ of essentially bounded measurable functions on a general measure 
space. Here Hölder's inequality and Minkowski's inequality are proved for $p \in(0, \infty]$. $\$ 15$ introduces the Banach space and its dual. $\S 16$ treats $L^{p}$ spaces for $p \in[1, \infty]$ as well as for $p \in(0,1) . \S 17$ treats relation among the $L^{p}$ spaces for different values of $p$. The $\ell^{p}$ spaces of sequences of numbers $\left(\alpha_{n}: n \in \mathbb{N}\right)$ with $\sum_{n \in \mathbb{N}}\left|\alpha_{n}\right|^{p}<\infty$ is treated as a particular case of $L^{p}$ spaces in which the underlying measure space is the counting measure space on the set $\mathbb{N}$ of natural numbers. The Riesz representation theorem on the $L^{p}$ spaces is proved in $\S 18$. $\$ 19$ treats integration on a locally compact Hausdorff space. Urysohn's Lemma on the existence of a continuous function with compact support and partition of unity, Borel and Radon measures, the Riesz representation theorem on the space of continuous functions with compact support as well as Lusin's theorem on approximation of a measurable function by continuous functions are included here. (The placement of $\S 19$ in Chapter 4 is somewhat arbitrary.)

Chapter 5 treats extension of additive set functions to measures. It starts with extension of an additive set function on an algebra to a measure in $\$ 20$ and completes the theory with extension of an additive set function on a semialgebra to a measure in $\S 21$. (Semialgebra of sets is an abstraction of the aggregate of left-open and right-closed boxes in the Euclidean space $\mathbb{R}^{n}$. Its importance lies in the fact that the Cartesian product of finitely many algebras and in particular $\sigma$-algebras is in general not an algebra, but only a semialgebra.) As an example of extending an additive set function on a semialgebra to a measure, the Lebesgue-Stieltjes measure determined by a real-valued increasing function on $\mathbb{R}$ is treated in $\$ 22$. Theorems establishing the equivalence of the absolute continuity and singularity of a Lebesgue-Stieltjes measure with respect to the Lebesgue measure with the absolute continuity and singularity of the increasing function that determines the Lebesgue-Stieltjes measure are proved. As a second example of extending an additive set function on a semialgebra to a measure, the product measure on the product of finitely many measure spaces is included in $\$ 23$. Tonelli's theorem and Fubini's theorem on the reduction of a multiple integral to iterated integrals are found here.

Chapter 6 specializes in integration in the Lebesgue measure space on $\mathbb{R}^{n}$. In $\$ 24$ the Lebesgue measure on $\mathbb{R}^{n}$ is constructed as an extension of the notion of volumes of boxes in $\mathbb{R}^{n}$ to Lebesgue measurable subsets of $\mathbb{R}^{n}$. Then it is shown that the Lebesgue measure space on $\mathbb{R}^{n}$ is the completion of the $n$-fold product of the Lebesgue measure space on $\mathbb{R}$. Regularity of the Lebesgue measure and in particular approximation of Lebesgue measurable sets by open sets leads to approximation of the integral of a measurable function by that of a continuous function. Translation invariance of the Lebesgue measure and integral and linear transformation of the Lebesgue measure and integral are treated. $\$ 25$ begins with the study of the average function of a locally integrable function. Hardy-Littlewood maximal theorem and Lebesgue differentiation theorem are presented. These are followed by differentiation of a set function with respect to the Lebesgue measure, in particular differentiation of a signed Borel measure with respect to the Lebesgue measure, and density of a Lebesgue measurable set with respect to the Lebesgue measure. $\$ 26$ treats change of variable of integration by differentiable transformations.

Chapter 7 is an introduction to Hausdorff measures on $\mathbb{R}^{n}$. $\$ 27$ defines $s$-dimensional Hausdorff measures on $\mathbb{R}^{n}$ for $s \in[0, \infty)$ and the Hausdorff dimension of a subset of $\mathbb{R}^{n}$. 
$\$ 28$ studies transformations of Hausdorff measures. $\$ 29$ shows that a Hausdorff measure of integral dimension is a constant multiple of the Lebesgue measure of the same dimension.

Every concept is defined precisely and every theorem is presented with a detailed and complete proof. I endeavored to present proofs that are natural and inevitable. Counterexamples are presented to show that certain conditions in the hypothesis of a theorem can not be simply dropped. References to earlier results within the text are made extensively so that the relation among the theorems as well as the line of development of the theory can be traced easily. On these grounds this book is suitable for self-study for anyone who has a good background in advanced calculus.

In writing this book I am indebted to the works that $I$ consulted. These are listed in the Bibliography. I made no attempt to give the origin of the theory and the theorems. To be consistent, I make no mention of the improvements that I made on some of the theorems. I take this opportunity to thank all the readers who found errors and suggested improvements in the various versions of the lecture notes on which this book is based.

Corona del Mar, California

J. Yeh

January, 2000 


\section{Preface to the Second Edition}

In this new edition all chapters have been revised and additional material have been incorporated although the framework and organization of the book are unchanged. Specifically the following sections have been added:

\section{$\S 13$ [VI] Length of Rectifiable Curves \\ $\S 15$ [VII] Baire Category Theorem \\ [VIII] Uniform Boundedness Theorem \\ [IX] Open Mapping Theorem \\ [X] Hahn-Banach Extension Theorems}

$\S 16$ weak convergence in $L^{p}$ spaces in [III] and [IV] of $\S 16$

the complete metric spaces $L^{p}$ for $p \in(0,1)$ in [V] of $\$ 16$

$\S 19$ [V] Signed Radon Measures

[VI] Dual Space of $C(X)$

$\$ 23$ [IV.2] Convolution of $L^{p}$ Functions

[IV.3] Approximate Identity in Convolution Product

[IV.4] Approximate Identity Relative to Pointwise Convergence

Besides these topics there are additional theorems in sections: $\S 1, \S 4, \S 5, \S 8, \S 10, \S 11$, $\S 13, \S 15, \S 16, \S 17, \S 19, \S 20, \S 21, \S 23, \S 24, \S 25$, and $\S 27$. Also 64 problems have been added.

To use this book as a textbook, selection of the following sections for instance makes a possible one-year course at the graduate level:

$$
\S 1 \text { to } \S 13, \S 15([\mathrm{I}] \text { to }[\mathrm{VI}]), \S 16 \text { to } \S 21, \S 23([\mathrm{I}] \text { to }[\mathrm{III}])
$$

It is my pleasure to thank Abel Klein for his helpful comments on the first edition of this book.

Corona del Mar, California

March, 2006 
This page is intentionally left blank 


\section{List of Notations}

$\mathbb{N}$

$\mathbb{Z}$

$\mathbb{Z}_{+}$

$\mathbb{R}$

$\mathbb{C}$

$\mathbb{K}$

$\overline{\mathbb{R}}$

$\overline{\mathbb{C}}$

$\mathbb{R}^{n}$

$\mathfrak{P}(X)$

$\mathfrak{B}_{X}$

$\mathfrak{B}_{\mathbb{R}}$

$\mathfrak{B}_{\mathbb{R}^{n}}$

$\left(\mathfrak{B}_{\mathbb{R}^{n}}\right)_{b}$

$\mathfrak{M}_{L}$

$\mathfrak{M}^{n}$

$\alpha(\mathfrak{E})$

$\sigma(\mathfrak{E})$

$\mathfrak{M}\left(\mu^{*}\right)$

$\mathfrak{A} \otimes \mathfrak{B}$

$\mathfrak{I}_{o}, \mathfrak{I}_{o c}, \mathfrak{I}_{c o}, \mathfrak{I}_{c}$ $\mathfrak{I}_{o}^{n}, \mathfrak{I}_{o c}^{n}, \mathfrak{I}_{c o}^{n}, \mathfrak{I}_{c}^{n}$

$\mathfrak{D}(f)$

$\mathfrak{R}(f)$

$\{D: f \leq \alpha\}$

$\mu_{L}^{*}$

$\mu_{L}$

$\left(\mu_{L}^{n}\right)^{*}$

$\mu_{L}^{n}$

$\left(\mathbb{R}, \mathfrak{M}_{L}, \mu_{L}\right)$

$\left(\mathbb{R}^{n}, \mathfrak{M}_{L}^{n}, \mu_{L}^{n}\right)$

$\left(\mathbb{R}, \mathfrak{M}\left(\mu_{g}^{*}\right), \mu_{g}\right)$

$\mathcal{L}^{p}(X, \mathfrak{A}, \mu)$

$L^{p}(X, \mathfrak{A}, \mu)$

$\mathcal{L}^{\infty}(X, \mathfrak{A}, \mu)$

$L^{\infty}(X, \mathfrak{A}, \mu)$

$\mathcal{L}_{\text {loc }}^{1}\left(\mathbb{R}^{n}, \mathfrak{M}_{L}^{n}, \mu_{L}^{n}\right)$ the natural numbers $\quad$ p.3

the integers p.3

the nonnegative integers p.3

the real numbers p.1

the complex numbers p.323

$\mathbb{R}$ or $\mathbb{C} \quad$ p.323

the extended real number system $\{-\infty\} \cup \mathbb{R} \cup\{\infty\} \quad$ p.11

the set of $\zeta=\xi+i \eta$ where $\xi, \eta \in \overline{\mathbb{R}} \quad$ p.376

the $n$-dimensional Euclidean space p.597

the collection of all subsets of a set $X$

p. 4

the $\sigma$-algebra of Borel sets in a topological space $X \quad$ D1.16, p.10

the $\sigma$-algebra of Borel sets in $\mathbb{R}$

the $\sigma$-algebra of Borel sets in $\mathbb{R}^{n}$

D1.16, p.10

D1.16, p.10

the collection of bounded Borel sets in $\mathbb{R}^{n} \quad$ D25.19, p.632

the $\sigma$-algebra of Lebesgue measurable sets in $\mathbb{R}$

the $\sigma$-algebra of Lebesgue measurable sets in $\mathbb{R}^{n}$

D3.1, p.41

D24.8, p.600

D1.12, p.7

D 1.12, p. 7

D2.2, p. 28

D23.3, p.528

D3.1, p.41

D24.2, p.597

p. 19

p. 19

p.70

D3.1, p.41

D3.1, p.41

Lebesgue measure on $\mathbb{R}$

Lebesgue outer measure on $\mathbb{R}^{n}$

Lebesgue measure on $\mathbb{R}^{n}$

D24.5, p.598

D24.8, p.600

D3.1, p.41

Lebesgue measure space on $\mathbb{R}$

D24.8, p.600

D22.5, p.507

D16.8, p.378

D16.22, p.385

D16.38, p.394

D16.42, p.395

D25.1, p.620 


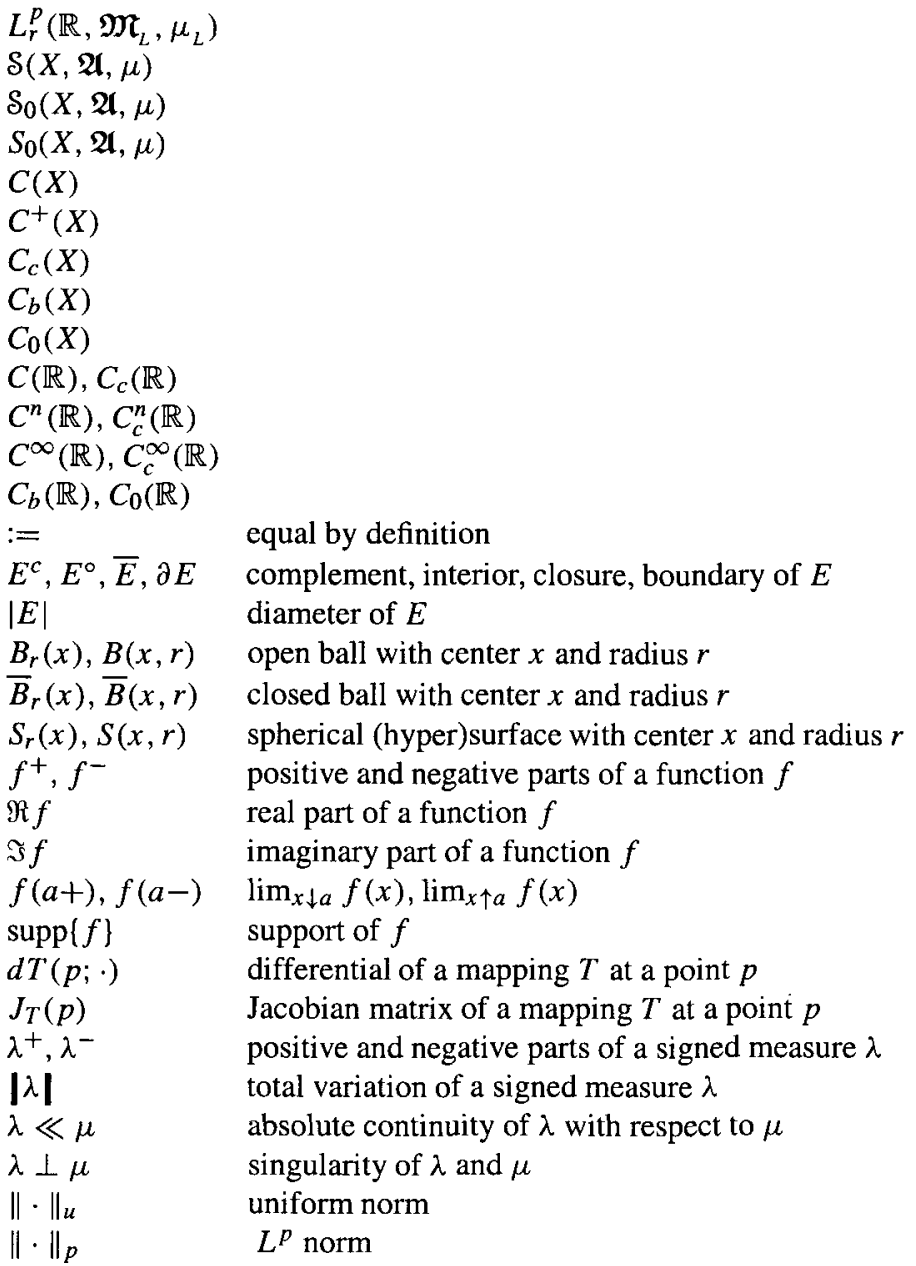

$\|\cdot\|_{\infty}$

$\|L\|_{V, W}$

$\|f\|_{*}$

$\mathcal{H}_{\delta}^{s}$

$\mathcal{H}^{s}$

$\mathcal{H}^{\bullet}(E)$

$\mathcal{F}_{\delta}^{s}, \mathcal{F}^{s}, \mathcal{G}_{\delta}^{s}, \mathcal{G}^{s}$

$\mathcal{S}_{\delta}^{s}, \mathcal{S}^{s}, \mathcal{B}_{\delta}^{s}, \mathcal{B}^{s}$

$\mathrm{e}_{\delta}^{s}, \mathrm{es}^{s}$

$\mathcal{K}_{\delta}^{s}, \mathcal{K}^{s}$

$\operatorname{dim}_{H}$
D23.29, p.547

L18.2, p.432

L18.2, p.432

T18.3, p.433

D19.48, p.471

D19.48, p.471

D19.9, p.447

D23.45, p.564

D23.45, p.564

D23.38, p. 555

D23.38, p. 555

D23.38, p. 555

D23.45, p. 564

D27.1, p.675

D15.33, p.338

D15.33, p.338

D15.33, p.338

D4.25, p.83

p. 376

p.376

p. 251

D19.7, p.447 p.649 p. 650

D10.22, p.213

D10.22, p.213

D11.4 , p. 225

D10.16, p.211

T19.50, p.471

D16.8, p.378

D16.36, p.393

D15.29, p.337

D15.39, p344

D27.3, p.675

D27.7, p.677 p. 688

T27.13, p.680

P27.14, p.681

T27.24, p.684

C27.25, p.685

D27.34, p.691 


\section{Euler Fraktur and Script}

Here is a list of the capital Roman letters, each followed by its corresponding Euler Fraktur and Script letters:
$A, \mathfrak{A}, \mathcal{A} \quad B, \mathfrak{B}, \mathcal{B}$
$C, \mathfrak{C}, \mathfrak{C}$
$D, \mathfrak{D}, \mathcal{D} \quad E, \mathfrak{E}, \mathcal{E}$
$F, \mathfrak{F}, \mathcal{F}$
$G, \mathfrak{G}, \mathcal{G}$
$H, \mathfrak{H}, \mathcal{H}$
$I, \mathfrak{I}, \mathcal{J}$
$J, \mathfrak{J}, \mathcal{J}$
$K, \mathfrak{K}, \mathcal{K}$
$L, \mathfrak{L}, \mathcal{L} \quad M, \mathfrak{M}, \mathcal{M}$
$N, \mathfrak{N}, \mathcal{N} \quad O, \mathfrak{O}, \mathcal{O}$
$P, \mathfrak{P}, \mathcal{P}$
$Q, \mathfrak{Q}, \mathcal{Q} \quad R, \mathfrak{R}, \mathfrak{R}$
$S, \mathfrak{S}, \mathbb{S}$
$T, \mathfrak{T}, \mathfrak{T} \quad U, \mathfrak{U}, \mathcal{U}$
$V, \mathfrak{V}, \mathcal{V}$
$W, \mathfrak{W}, \mathcal{W} \quad X, \mathfrak{X}, X$
$Y, \mathfrak{Y}, y \quad Z, \mathfrak{z}, Z$ 